\title{
PERCEPÇÃO DOS FAMILIARES DE USUÁRIOS DE SUBSTÂNCIAS PSICOATIVAS EM RELAÇÃO AO TRATAMENTO EM COMUNIDADE TERAPÊUTICA*
}

Genézi Guedes da Silva ${ }^{1}$, Melissa Ricardo Pinto ${ }^{2}$, Gicelle Galvan Machineski ${ }^{2}$

\begin{abstract}
RESUMO: O objetivo deste estudo foi conhecer a percepção dos familiares de usuários de substâncias psicoativas em relação ao tratamento realizado. Para tanto, uma pesquisa qualitativa e descritiva foi realizada em uma Comunidade Terapêutica de Cascavel, Paraná, com 10 familiares de usuários, no período de julho a agosto de 2012. As informações foram analisadas por meio da técnica de análise de conteúdo com identificação das categorias Tempo de permanência na Comunidade Terapêutica; Insegurança e medo como sentimentos expressos, Perspectiva de difícil convivência familiar; e Satisfação relativa à metodologia proposta. Compreendemos que o familiar necessita da instrumentalização dos profissionais envolvidos no cuidado para a reabilitação social dos usuários.
\end{abstract}

DESCRITORES: Enfermagem; Família; Comunidade terapêutica.

\section{THE PERCEPTION OF FAMILY MEMBERS OF USERS OF PSYCHOACTIVE SUBSTANCES IN RELATION TO TREATMENT IN A THERAPEUTIC COMMUNITY}

ABSTRACT: This study aimed to investigate the perception of the family members of psychoactive substance users in relation to the treatment undertaken. To this end, qualitative and descriptive research was undertaken in a Therapeutic Community in Cascavel, in the state of Paraná, with 10 family members of users, in the period July - August 2012. The information was analyzed using the technique of content analysis, identifying the categories Length of stay in the Therapeutic Community; Insecurity and fear as feelings expressed; The perspective of difficulty in family coexistence; and Satisfaction relative to the methodology proposed. We understand that the family member needs the instrumentalization of the professionals involved in the care for the users' social rehabilitation.

DESCRIPTORS: Nursing; Family; Therapeutic community.

\section{PERCEPCIÓN DE LOS FAMILIARES DE USUARIOS DE SUSTANCIAS PSICOACTIVAS ACERCA DEL TRATAMIENTO EN COMUNIDAD TERAPÉUTICA}

RESUMEN: El objetivo de este estudio fue conocer la percepción de los familiares de usuarios de sustancias psicoactivas acerca del tratamiento realizado. Para eso, una investigación cualitativa y descriptiva fue realizada en una Comunidad Terapéutica de Cascavel, Paraná, con 10 familiares de usuarios, en el periodo de julio a agosto de 2012. Las informaciones fueron analizadas por medio de la técnica de análisis de contenido con identificación de las categorías Tiempo de permanencia en la Comunidad Terapéutica; Inseguridad y miedo como sentimientos expresos, Perspectiva de difícil convivencia familiar; y Satisfacción acerca de la metodología propuesta. Se comprende que el familiar necesita da instrumentalización de los profesionales involucrados en el cuidado para la rehabilitación social de los usuarios.

DESCRIPTORES: Enfermería; Familia; Comunidad terapéutica.

*Artigo resultante de Trabalho de Conclusão de Curso apresentado ao Curso de Enfermagem da Faculdade Assis Gurgacz em 2012.

${ }^{1}$ Enfermeira.

${ }^{2}$ Enfermeira Doutora em Enfermagem. Professora do Curso de Enfermagem da Faculdade Assis Gurgacz.

Autor correspondente:

Recebido: 30/11/2012

Gicelle Galvan Machineski

Aprovado: 03/06/2013

Faculdade Assis Gurgacz

Rua Manaus, 2046 - 85811-030 - Cascavel-PR-Brasil

E-mail: gicelle@fag.edu.br 


\section{INTRODUÇÃO}

Durante o Curso de Graduação em Enfermagem visualizamos que o profissional enfermeiro está inserido no meio social, trabalhando com a comunidade em geral. Sendo assim, entende-se que ele esteja envolvido na prevenção, no tratamento e no processo de reabilitação dos usuários de substâncias psicoativas. O referido tratamento pode ser oferecido em serviços substitutivos em saúde mental, como unidade básica de baúde, o hospital dia, os leitos psiquiátricos em hospitais gerais, os centros de atenção psicossocial e as comunidades terapêuticas.

Este estudo aborda, especificamente, o tratamento realizado em uma comunidade terapêutica e tem como objetivo conhecer a percepção dos familiares em relação ao tratamento realizado. Nesse contexto, o papel da família é fator determinante tanto no ciclo em que o usuário se encontra na comunidade terapêutica, quanto no período de sua reinserção social. Pois, o ambiente emocional familiar tem grande influência no processo de recuperação do indivíduo, sendo necessária uma rede de apoio à família e ao dependente químico no pós-tratamento.

Por essas questões, acreditamos na importância da realização do estudo buscando o entendimento da problemática relativa à família e aos usuários, com o propósito de construir propostas consistentes para seu atendimento. Nessa perspectiva, com o intuito de contextualizar a assistência oferecida aos usuários de substâncias psicoativas, a seguir discorremos acerca da evolução histórica da saúde mental no Brasil.

Dados do Ministério da Saúde ${ }^{(1)}$ destacam que, ao longo da história, a loucura era vista como um fenômeno sobrenatural e as instituições para loucos tinham a finalidade de afastar determinados indivíduos do convívio dos considerados normais. Dessa forma, os indivíduos em sofrimento psíquico eram hospitalizados em locais distantes das cidades, onde ficavam excluídos do convívio social e familiar.

O modelo manicomial no Brasil foi superado a partir de políticas de saúde, por meio de discussões nas conferências de saúde mental que foram realizadas no decorrer dos anos. O marco histórico de tal superação é a VIII Conferência Nacional de Saúde, que aconteceu em 1986, e o processo de elaboração da nova Constituição de 1988, no qual o movimento sanitário e o governo reencontram-se com os movimentos sociais populares, inaugurando-se uma nova fase de participação social nas Políticas Públicas de
Saúde. Nesse contexto, a Reforma Psiquiátrica brasileira teve grande influência dos movimentos que efervesciam na Europa, em específico a Psiquiatria Democrática Italiana, cujo objetivo principal era reinventar o modelo de assistência. Tal movimento tinha na comunidade e nas relações que ela estabelece com o louco a base para a transformação do modelo psiquiátrico $^{(2)}$. Assim, abriu-se espaço para serviços substitutivos de tratamentos, tais como a comunidade terapêutica, tido como modelo desidencial e cujo termo teve como pioneiro Maxwell Jones - é oriundo de hospitais psiquiátricos, em que a responsabilidade do tratamento era dividida entre o médico e os demais membros da comunidade. A partir de 1953, desenvolveu-se, nos ambientes hospitalares, um lugar organizado em que se esperava que todos contribuíssem ativamente para metas comuns no processo de cura, modificando terapeuticamente uma pessoa ${ }^{(3)}$.

No decorrer das décadas, surgiram outros movimentos, na grande maioria, formados por grupos religiosos, que se utilizavam do modelo da comunidade terapêutica como forma de tratamento ${ }^{(4)}$. Nos dias atuais, as comunidades terapêuticas têm exercido importante no tratamento psicossocial de usuários de substâncias psicoativas no Brasil. Com o crescente aumento do consumo de drogas no decorrer dos últimos anos, a expansão dessas comunidades aumentou, significativamente, no país. Assim, para regulamentar o funcionamento das Comunidades Terapêuticas, a Agência Nacional de Vigilância Sanitária (ANVISA) editou a RDC n. 29, de 30 de junho de $2011^{(5)}$, a qual norteia todo o trabalho realizado dentro das Comunidades Terapêuticas.

Contudo, percebe-se a importância do envolvimento e a participação dos profissionais nos programas de assistência aos usuários de substâncias psicoativas, tanto no ensino como em novas pesquisas, de forma a aprimorar os conhecimentos para melhor atender essa clientela, visto que

monitorar a saúde mental na comunidade, dar mais apoio a Pesquisa e estabelecer programas específicos são práticas que devem ser obrigatoriamente contempladas pela Política de Atenção a Usuários de álcool e outras Drogas, em uma perspectiva ampliada de Saúde Pública ${ }^{(6: 11)}$.

No contexto atual brasileiro, a dependência química atinge milhares de pessoas, contribuindo de maneira elevada no assustador índice de criminalidade. Segundo a Classificação de Transtornos Mentais e

Cogitare Enferm. 2013 Jul/Set; 18(3):475-81 
de Comportamento, apontada na Classificação Internacional de Doenças (CID-10), uma característica descritiva central da síndrome de dependência é o desejo (frequente, forte e algumas vezes irresistível) de consumir drogas psicoativas, álcool ou tabaco ${ }^{(7)}$.

Nesse sentido, a dependência química é tratada em serviços substitutivos em saúde mental, os quais incluem os familiares no tratamento. Assim, os familiares são convidados a participar do serviço substitutivo, a fim de que se estabeleça uma relação entre família, usuário e serviço ${ }^{(8)}$. Esse envolvimento é importante para que a intervenção seja eficaz, pois a família tem grande influência no processo de recuperação, podendo contribuir eficazmente para o desenvolvimento do bem-estar físico, mental e social do usuário.

Diante dessa situação, percebemos a necessidade de um planejamento de ações preventivas e relativas ao uso de álcool e outras drogas. Conforme o Conselho Nacional Antidrogas (CONAD) estabelece, na Resolução n. 3/2005, é necessário

educar, informar, capacitar e formar pessoas em todos os segmentos sociais para a ação efetiva e eficaz de redução da demanda, da oferta e de danos, fundamentada em conhecimentos científicos validados e experiências bem-sucedidas, adequadas à nossa realidade ${ }^{(9: s / p)}$.

Assim, percebe-se que a dependência química é uma questão de saúde pública. Nesse sentido, é relevante trabalhar no fortalecimento dessas políticas e instrumentar os familiares para que se sintam mais seguros em conviver com aquele que sofre pelo uso de álcool e outras drogas, incluindo ações que visem ao cuidado geral com a saúde e a reinserção psicossocial do usuário. O objetivo desta pesquisa foi conhecer a percepção dos familiares de usuários de substâncias psicoativas em relação ao tratamento realizado na Comunidade Terapêutica.

\section{MÉTODO}

Trata-se de um estudo qualitativo, descritivo, realizado em uma Comunidade Terapêutica do Município de Cascavel, Estado do Paraná, com 10 familiares de usuários de substâncias psicoativas que estavam em tratamento devido ao uso de álcool e outras drogas, sendo que, entre os participantes, seis eram do sexo feminino e quatro do sexo masculino.

Foram incluídos na pesquisa os familiares que mais conviviam com os usuários da comunidade terapêutica e aceitaram participar do estudo mediante a assinatura do Termo de Consentimento Livre e Esclarecido.

A coleta das informações aconteceu entre os meses de julho e agosto de 2012, após a aprovação do estudo pelo Comitê de Ética em Pesquisa da Faculdade Assis Gurgacz, cumprindo o proposto pela Resolução n. 196/96 do Conselho Nacional de Saúde ${ }^{(10)}$, sob parecer n. 117/2012. A técnica utilizada para esse fim foi a entrevista semiestruturada, com as seguintes questões norteadoras: Como você se sente ao pensar no retorno do seu familiar ao convívio domiciliar?; $\mathrm{O}$ que você acha da metodologia utilizada na Comunidade Terapêutica?; e Em relação ao programa proposto e à equipe, qual sua satisfação?. As informações coletadas foram gravadas e posteriormente transcritas na íntegra, sendo analisadas a partir da técnica de análise de conteúdo ${ }^{(11)}$. Com a finalidade de garantir $o$ anonimato, os familiares foram identificados com letras do alfabeto, de acordo com a ordem em que aconteceram as entrevistas.

\section{RESULTADOS}

A partir da análise, emergiram as seguintes categorias apresentadas com respectivos recortes de depoimentos.

\section{Tempo de permanência na comunidade terapêutica}

[...] era tratada com medicamentos e ficava só trinta dias e aqui ela fica mais tempo, mais natural. Fica mais consciente do que está fazendo, sobre o tratamento que ela está fazendo, e o tratamento que está recebendo. (A)

[...] não estou falando que não seja viável o tratamento em hospital psiquiátrico, mas apenas para desintoxicar. Depois, eles precisam de tratamento mais prolongado, como o que é feito na Comunidade Terapêutica. (I)

\section{Insegurança e medo como sentimentos expressos e difícil convivência familiar}

Eu penso que é uma preocupação como vai ser a convivência dela lá com a família porque não são todos que compreendem a situação da pessoa[...]. Alguns querem forçar a pessoa a se libertar da de- 
pendência né, e não é por esse rumo, esse meio. (A) [...] porque não tem condições de eu confiar mais nele, a gente não tem mais confiança, eu não posso assim confiar nele porque se ele falar que vai ao mercado comprar alguma coisa e ele demora eu fico ansiosa, e já fico pensando que ele já foi usar droga de novo. (D)

\section{Satisfação relativa à metodologia proposta}

Eu acho que a forma que eles trabalham é positiva, o tratamento deles, porque nos outros tratamentos que ele fez, quando a gente chegava lá, dava uma tristeza de ver ele. Os remédios deixavam ele meio abobado; não é que eles não cuidassem bem, é que um tratamento muito forte, porque não adianta só tomar os remédios, o que manda é o carinho é o amor [...]. (E)

Eu não tenho um vasto conhecimento do método que eles têm de tratar, eu só sei que lá eles têm a parte religiosa, espiritual que isso ajuda e muito [...].Outro ponto positivo que eu acho é o fato de não ter aquele exagero de medicamentos, isso é bom. Eu acho que eles estão fazendo um trabalho bom, tá dando certo [...]. (H)

\section{DISCUSSÃO}

Ao analisarmos a compreensão dos familiares em relação ao tratamento realizado na Comunidade Terapêutica, percebemos que esses indivíduos concebem o tempo de permanência na instituição como um fator que contribui para a recuperação, comparado a tratamentos, já realizados em outras clínicas, com tempo de permanência menor.

A partir das colocações, percebemos que, após o usuário passar por vários tratamentos e não alcançar o sucesso esperado na recuperação, os familiares esperam encontrar na Comunidade Terapêutica uma chance de ter o usuário livre da dependência química.

Nesse sentido, compreendemos que

quando os familiares buscam atendimento [...] visam a obter tratamento ao seu familiar usuário, tendo em vista a melhora do seu quadro clínico com remissão de sintomas e melhorias na interação $\operatorname{social}^{(12: 131)}$.

Cabe esclarecer que os tratamentos realizados em clínicas especializadas para fins de desintoxicação não visam lidar com os problemas sociais e comportamentais associados ao uso de drogas, consequentemente não produzem a mudança de comportamento neces- sária para a recuperação e esperada pelos familiares, de modo que há a demanda de uma continuidade após esse período.

Assim, tem-se, como uma das alternativas, o tratamento realizado na Comunidade Terapêutica, que propõe, por meio da aplicação de sua metodologia, trabalhar nesse âmbito, desde que o usuário aceite livremente participar das atividades propostas, previamente informadas a ele e a seus familiares.

Tal serviço substitutivo tem como característica ser uma opção de tratamento à margem das correntes tradicionais. Quando os princípios de recuperação, resgate da cidadania, reabilitação física e psicológica e reinserção social são aplicados satisfatoriamente, o tratamento apresenta bons resultados, agindo em fatores psicossociais da dependência ${ }^{(13)}$.

Nesse sentido, é importante trabalhar com os familiares, esclarecendo o compromisso que eles têm com o usuário, pois muitas vezes há acomodação diante da realidade, de forma que falta interação nas possíveis mudanças no ambiente familiar, as quais são necessárias para o sucesso da recuperação do indivíduo em tratamento. Faz-se necessário compreender que

\begin{abstract}
a família perto participando de todo o processo que o tratamento venha exigir, faz com que o dependente químico venha a perceber que ainda há possibilidades, ainda há diálogo, a família não desistiu e que ainda acredita na eficácia do tratamento ${ }^{(14: 62)}$.
\end{abstract}

Isso evidencia o quanto é significante para o dependente em recuperação o acompanhamento da família durante o período em que se encontra na Comunidade Terapêutica. Da mesma maneira, a partir do momento do retorno à convivência familiar, é essencial ao indivíduo receber os cuidados e o apoio.

Outro aspecto que verificamos nesse estudo é que o ambiente familiar é modificado com a presença do usuário de substâncias psicoativas, as relações com as famílias sofrem alterações, bem como as relações com o mundo externo.

[...] é desgastante para a família conviver com o sofrimento psíquico, implicando compreender e lidar com comportamentos não-convencionais [...], os quais terminam por despertar sentimentos ambíguos $[. . .]^{(15: 590)}$.

Quando os familiares foram questionados sobre o retorno do usuário ao convívio familiar, expressa- 
ram sentimentos de medo, insegurança e perspectiva de difícil convivência. $\mathrm{O}$ medo e a desconfiança que existem dificultam o relacionamento entre os membros da família, e nos parece que a inserção e participação desses familiares no processo de recuperação do usuário ocorrem de forma ainda muito superficial. Isso traz como consequência insegurança, muitas vezes gerada pela falta de envolvimento efetivo no tratamento, pois se desconhecem as mudanças ocorridas com o indivíduo e suas reais necessidades para o momento. Assim "tem sido consenso, entre especialistas em drogadição, a necessidade de participação da família no tratamento e no manejo dos problemas do usuário de drogas"(16:52).

Faz-se evidente a importância de trabalhar junto a essas famílias no intuito de instrumentar no manejo com o usuário quando do retorno ao convívio familiar, contribuindo no fortalecimento das relações familiares e sociais, conforme mencionado em estudos referentes ao tema.

Além do mais, nesse contexto:

o cuidado familial caracteriza-se pelas relações e interações sociais que a família desenvolve e [...] fortalece-se a partir da rede de suporte social formada por parentes, amigos e vizinhos ${ }^{(8: 42)}$.

Tal colocação enfatiza quão importante se faz uma rede estruturada para ajudar o indivíduo que se encontra em processo de recuperação. Desse modo, é necessário trabalhar a origem dos problemas com drogas, pois o abuso dessas substâncias é consequência da atual configuração da sociedade e o comportamento dos pais afeta o dos filhos e vice-versa ${ }^{(17)}$.

Em relação à metodologia utilizada na Comunidade Terapêutica, os entrevistados demonstram satisfação, apesar da insegurança acerca do retorno do usuário ao convívio familiar. A respeito de tal questão, não podemos deixar de comentar a importância de Políticas Públicas bem articuladas, a serem asseguradas de forma integral, enfatizando a importância de um trabalho em rede no pós-tratamento, como está estabelecido na política do Ministério da Saúde de Atenção integral ao usuário de álcool e outras drogas ${ }^{(6)}$, que tem nas diretrizes do SUS seus eixos centrais, sob os quais trabalha as especificidades de seu público-alvo.

O uso de álcool e outras drogas, por tratar-se de um tema transversal a outras áreas da saúde, da justiça, da educação, social e de desenvolvimento, requer intensa capilaridade para a execução de políticas de atenção integral ao consumidor de álcool e outras drogas ${ }^{(6: 20)}$.
Assim, podemos entender a importância do estabelecimento de vínculos e da construção da responsabilidade pelo tratamento, por meio da mobilização da sociedade civil, da oferta de condições e da participação nas práticas preventivas terapêuticas e reabilitadoras.

Além disso, é imprescindível a disponibilização de acesso a programas voltados para geração de renda, profissionalização e participação comunitária, por exemplo, de modo a garantir a recuperação integral. Nesse aspecto, se coloca

a necessidade de os órgãos governamentais instituírem mais serviços extra-hospitalares que atendam a demanda populacional, fornecendo uma assistência de qualidade, para que haja o aumento de adesão dos dependentes químicos a eles ${ }^{(18: 450)}$.

Compreendemos que os familiares avaliaram positivamente o tratamento realizado na instituição, apesar de se sentirem despreparados para o retorno da pessoa em recuperação ao convívio familiar,

nesse sentido, a família precisa de apoio de uma equipe multiprofissional que compõe os serviços substitutivos em saúde mental, a fim de entender as condições de seus membros e aprender a lidar com as situações que se impõem na interação com o indivíduo em sofrimento psíquico ${ }^{(19: 666)}$.

Cabe também aos profissionais sanar essa lacuna expressa pelos familiares, contribuindo, efetivamente, em novas possibilidades de assistência à família, por meio da promoção de mudanças sociais e da utilização de um sistema integrado e articulado em redes de saúde, com grande diversidade de pontos de atenção, voltadas às condições específicas de cada indivíduo.

Assim, a integração e a colaboração entre os diferentes atores em termos de focar nas co-morbidades físicas e mentais, bem como o trabalho intersetorial, incluindo moradia, educação, desenvolvimento e ambiente, podem aumentar a chance de que as aspirações em saúde mental se tornem realidade ${ }^{(20)}$.

\section{CONSIDERAÇÕES FINAIS}

A realização deste estudo - que teve como objetivo conhecer a percepção dos familiares de usuários de substâncias psicoativas em relação ao tratamento realizado na Comunidade Terapêutica - possibilitou-nos a compreensão de que os familiares reconhecem a importância do tratamento técnico e humano ofere- 
cido naquele serviço, apesar de alguns entrevistados desconhecerem o programa proposto pela instituição e sua metodologia. Tal desconhecimento descortina uma lacuna importante, pois evidencia certo distanciamento quanto ao tratamento de seu familiar, não contemplando o que anteriormente discutimos sobre a importância do envolvimento dos familiares no tratamento do dependente em recuperação.

Quando questionados acerca do retorno ao convívio familiar, os entrevistados expressaram sentimentos de medo e insegurança, apontando para uma difícil convivência familiar. Esse fato indica a imprescindibilidade de que os profissionais, principalmente da enfermagem, se envolvam no cuidado e instrumentem as famílias para o momento da reabilitação social. Percebe-se, também, a necessidade de se estabelecer uma rede de apoio no intuito de reinserir o indivíduo em recuperação na sociedade e no mercado de trabalho, garantindo-lhe assim atendimento integral.

Nesse sentido, a enfermagem elabora, junto aos usuários da comunidade terapêutica e seus familiares, planos terapêuticos individuais em conjunto com uma equipe multidisciplinar a fim de estabelecer as necessidades primordiais e orientar a família quanto à forma de relacionar-se com seus membros no momento do retorno ao lar. Em relação à referida rede de apoio, são necessários programas de reinserção social do usuário no sentido de ser empregado e continuar seu tratamento a fim de evitar ou atender possíveis recaídas.

Para finalizar, é importante esclarecer que o assunto proposto tem muito ainda a ser explorado, de forma que sejam possíveis novos conhecimentos e melhorias no atendimento à família e ao usuário.

\section{REFERÊNCIAS}

1. Ministério da Saúde (BR). Reforma psiquiátrica e política de saúde mental no Brasil. Conferência Regional de Reforma dos Serviços de Saúde Mental: 15 anos depois de Caracas. Brasília: Ministério da saúde; 2005.

2. Amarante PDC. O homem e a serpente. Outras histórias para a loucura e a psiquiatria. $20^{\mathrm{a}}$ ed. Rio de Janeiro: Fiocruz; 2010.

3. De Leon G. A comunidade terapêutica: teoria, modelo e método. São Paulo: Loyola; 2003.

4. Fracasso L. Comunidade Terapêutica: uma abordagem psicossocial. In: Anais do Encontro Interdisciplinar: dependência química, saúde e responsabilidade social, educando e transformando através da educação física; 2008. P. 10-20; Campinas, Brasil. Campinas; 2008.

5. Ministério da Saúde (BR). Agência Nacional de Vigilância Sanitária. Resolução da Direção Colegiada n. 29, de 30 de junho de 2011. Brasília; 2011.

6. Ministério da Saúde (BR). Secretaria de Atenção à Saúde. Política para a atenção integral a usuários de álcool e outras drogas. Brasília: Ministério da Saúde; 2004.

7. Organização Mundial da Saúde. Classificação de transtornos mentais e de comportamento da CID-10. Porto Alegre: Artes Médicas; 1993.

8. Machineski GG. O tipo vivido de familiares de usuários de um Centro de Atenção Psicossocial Infantil [tese]. Porto Alegre (RS): Universidade Federal do Rio Grande do Sul; 2011.

9. Ministério da Justiça (BR). Conselho Nacional Antidrogas. Política Nacional Sobre Drogas. Resolução n. 03, de 27 de outubro de 2005. Brasília; 2005.

10. Ministério da Saúde (BR). Conselho Nacional de Saúde. Resolução n. 196, de 14 de maio de 1996. Brasília; 2012.

11. Bardin L. Análise de Conteúdo. Trad. de Luis Ântero Reto e Augusto Pinheiro. Lisboa: Edições 70; 2004.

12. Machineski GG, Scnheider JF, Camatta MW. O tipo vivido de familiares de usuários de um centro de atenção psicossocial infantil. Rev. Gaúcha Enferm. [Internet]. 2013;34(1) [acesso em 28 maio 2013]. Disponível: http:// dx.doi.org/10.1590/S1983-14472013000100016

13. Raupp LM, Milnitiski-Sapiro C. A "reeducação" de adolescentes em uma comunidade terapêutica: o tratamento da drogadição em uma instituição religiosa. Psic:. Teor. e Pesq. 2008;24(3):361-8.

14. Maçaneiro A. Percepção do dependente químico quanto ao processo de recuperação [Projeto de Trabalho de Conclusão de Curso]. Itajaí (SC): Universidade do Vale do Itajaí; 2008.

15. Borba LO, Schwartz E, Kantorski LP. A sobrecarga da família que convive com a realidade do transtorno mental. Acta Paul. Enferm. 2008;21(4):588-94.

16. Silva JL. Programa de tratamento e prevenção para dependentes de drogas em Comunidade Terapêutica [tese]. São Paulo (SP): Faculdade de Saúde Pública da Universidade de São Paulo; 2011. 
17. Associação Brasileira de Amor Exigente. O que é Amor Exigente [Internet] [local desconhecido]: O que é Amor Exigente; 2012 [acesso em 14 set 2012]. Disponível: http://www.amorexigente.org.br/. [Não entendi essa referência, não soube arrumar]

18. Ferreira A, Capistrano F, Maftum M, Kalinke L, Kirchhof A. A caracterização de internações de dependentes químicos em uma unidade de reabilitação. Cogitare enferm. [Internet] 2012;17(3) [acesso em 26 nov 2012]. Disponível: http://ojs.c3sl.ufpr.br/ojs2/index. php/cogitare/article/view/29284/19033.

19. Machineski GG, Schneider JF, Bastos CCBC. The significance of having family members assisted in a capsi: a phenomenological study. Online Braz J Nurs. [Internet] 2012;11(3) [acesso em 28 maio 2013]. Disponível: http://www.objnursing.uff.br/index.php/ nursing/article/view/3788

20. Ivbijaro G. Mental health: the aspiration to reality gap. Ment Health Fam Med. [Internet] 2011;8(2) [acesso em 28 maio 2013]. Disponível: http://link.periodicos. capes.gov.br/sfxlc141?url_ver=Z39.88-2004\&url ctx_fmt=infofi/fmt:kev:mtx:ctx\&ctx_enc=info:ofi/ enc:UTF8\&ctx_ver=Z39.882004\&rfr_id=info:sid/ sfxit.com:azlist\&sfx.ignore_date_threshold=1\&rft. object_id=1000000000388956\&svc.fulltext=yes 